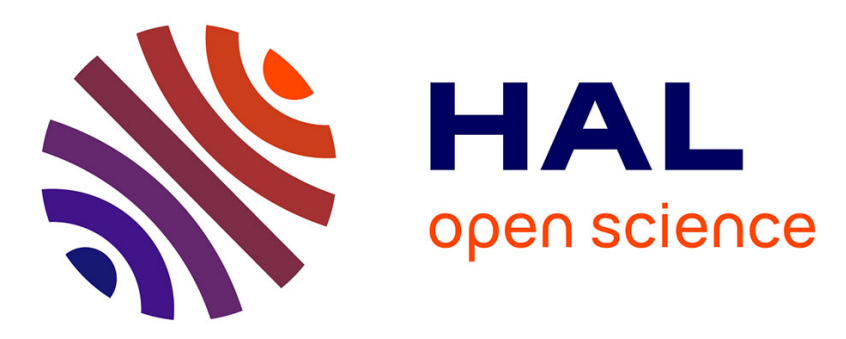

\title{
Visualising time with multiple granularities: a generic framework.
}

\author{
Iwona Dudek, Jean-Yves Blaise
}

\section{To cite this version:}

Iwona Dudek, Jean-Yves Blaise. Visualising time with multiple granularities: a generic framework.. 40th annual conference of Computer applications and quantitative methods in archaeology, Mar 2013, Southampton, United Kingdom. pp.470-481. halshs-01002275

\section{HAL Id: halshs-01002275 https://shs.hal.science/halshs-01002275}

Submitted on 5 Jun 2014

HAL is a multi-disciplinary open access archive for the deposit and dissemination of scientific research documents, whether they are published or not. The documents may come from teaching and research institutions in France or abroad, or from public or private research centers.
L'archive ouverte pluridisciplinaire HAL, est destinée au dépôt et à la diffusion de documents scientifiques de niveau recherche, publiés ou non, émanant des établissements d'enseignement et de recherche français ou étrangers, des laboratoires publics ou privés. 


\title{
Visualising time with multiple granularities: a generic framework. I.Dudek, JY Blaise, CNRS UMR CNRS/MCC 3495 MAP
}

\begin{abstract}
When investigating the evolution of historic architecture, and putting together various pieces of information (each with its specific characteristics in terms of precision, scope and reliability), time points and intervals the analyst will identify are often inconsistent in terms of granularity. Our contribution introduces graphic solutions that combine multiple aspects of the parameter time, and particularly multiple granularities. As a first step, we initially propose a visual comparison of 25 alternative calendars, covering a wide range of historic periods and cultures or civilisations.This first result is then extended to propose a more generic framework for visualising time with multiple granularities. It is applied on two very different test cases.

The contribution will present the concepts and ideas behind this research, as well as their practical applications on the tests cases and accordingly it possible benefits for researchers and practitioners in historic sciences.
\end{abstract}

\section{Introduction}

Investigating the evolution of historic artefacts most often starts with the cumbersome task of putting together various pieces of information, each with its specific characteristics in terms of precision, scope and reliability. Naturally, time slots are among the main clues analysts expect to spot when filtering and cross-examining these pieces of information.

In order to proceed to any type of reasoning (teleological or causal) one has to place all the data and pieces of information. But due to the very nature of historic data sets - heterogeneity, uncertainty, missing data, uneven distribution in time (etc.) - time points and intervals the analyst will identify are often inconsistent in terms of granularity. Time intervals, typically the overall lifetime of an artefact, its periods of construction or modification, may be described by expressions like "between the last quarter of the XIIIth $c$. and the middle of the XIVth c.", whereas some punctual events may be recorded more precisely, in cases like "the fire that occurred at night on Nov 29 $9^{\text {th }} 1554$ " or "the town's siege between March 1445 and November 1445".

In parallel, describing an artefact's life evolution often implies taking into consideration pieces of information that correspond to regular or cyclic events, with here also inconsistent granularities. Typically, when analysing an isolated chapel at high altitude, the analyst will need to cope with a fuzzy cyclic behaviour - the chapel is inaccessible due to snow for a certain number of weeks during the year - as well as with a well-defined cyclic behaviour - a pilgrimage is organised on the saint's day every year.

In other words, may it be because of the nature of historic data sets, or may it be because of the heterogeneity of the events we need to report, there are very few solutions analysts can count on if they need to visualise in a consistent, insight-gaining manner the time slots they have spotted.

In historic sciences it appears clearly that the handling of multiple time granularities is one of the major bottlenecks in the analyst's visualisation effort. Naturally, conveying properly doubts is an even harder challenge - with numerous overlapping issues, as will be shown. Our research aims at giving analysts means to combine in a single visualisation multiple aspects of the parameter time, and particularly multiple granularities.

As a first exploratory step, we initially focused on a visual comparison of 25 alternative calendars covering a wide range of historic periods and cultures or civilisations (Julian and Gregorian calendars of course, but also Babylon, ancient Egypt, China and Japan, Incas, Inuits, Burma and Bali, etc.). The visualisation sums up in a synthetic way key aspects of calendars (cycles, divisions, period of validity, area of validity, the intercalation - i.e. correction mechanisms, etc.) corresponding to alternative time granularities. The visualisation helps underlining legacies in between periods and areas, alternative visions of time as linear or cyclic, common or opposing choices like solar/lunar/lunisolar, alternative divisions of the year, mechanisms to cope with intercalations, etc.. A prominent service offered by this visualisation is that it enabling comparisons at various time granularities, "within the eyespan", to quote E.R Tufte. This first result has been extended to propose a more generic framework for visualising time with multiple granularities. It is applied on two test cases representing different scales and territories. The contribution will present the concepts and ideas behind this research, as well as their practical applications on the tests cases and accordingly it possible benefits for researchers and practitioners in historic sciences. 


\section{Research context}

Reasoning on the historic artefacts requires a deeper understanding of the time parameter than what we understand of it in everyday life. And if in addition we want to use visualisations to inquire into what is behind our time-oriented data, we need even more extensive understanding, analysis and control over the time parameter.

In parallel, Information Visualisation and Visual Analytics are multidisciplinary fields that are increasingly applied as a critical component in scientific research where visual reasoning is a relevant method (i.e. analytical method that employs human perception in order to inform, reveal unknowns or as a part of the thinking process itself (MacEachren et al.2005,139-160)). In other words visualisation is about: ... pictures of numbers ... pictures of nouns ... pictures of verbs, the representation of mechanism and motion, of process of dynamics, of causes end effects, of explanation and narrative... (Tufte1997, 126).

This is probably the reason why the time parameter has been carefully studied and structured notably in and around the above fields - see for example (Tufte 1990, 151), (Chardonnel 2007) (Knight 1993, 401-419), (Perlata 2004, 241-248), (Matoušek 2007, 239-254), (Allen 1984, 124-154) - although it doesn't mean that we at this stage fully understand its nature.

As demonstrated in (Aigner et al. 2008, 47-60) multiple aspects of time can be taken into account : time progression (linear time vs. cyclic time), time structure (ordered time, branching time, time with multiple perspectives), temporal entities and their relations (time points, time intervals), temporal scale (ordinal time, discrete time, continuous time, discontinuous time), time granularity, uncertainty of temporal position of temporal entities, etc..

However as sagely remarked "In theory, there is no difference between theory and practice. But in practice, there is. (...) Theory and practice both have limitations. Sometimes, practice proves the value of a good theory ..." (West 2010).

\subsection{Theory $v s$. practice}

Theoretical approaches are crucial in understanding the time parameter, still they are not the only solution to the problem. Another approach can be observing facts: here, historic calendars.

Calendars are basically an effort to position oneself in time, by observing and measuring objectively one or several phenomena that occur repeatedly, on a regular basis - may it be the course of planets, hunting seasons, market days in the neighbouring cities. In other words, understanding calendars means understanding how time is seen by a society, in a given space. This way calendars tell us something about societies, but in addition they also demonstrate the complexity of the time parameter, with several repetitive cycles to combine in a way that has to be understood by everyone (night \& day, seasons \& years, cultural events, etc.).

Finally, calendars are used not only to position oneself in absolute time, but also to position oneself with regards to other specific temporal slot, may they be "how many months since my last check-up at the dentist" or less depressing "how many grand-fathers since Copernic's discovery"? (account in grandfathers, as a tribute to T. Pratchett). Naturally this applies both to past and to future events. Accordingly calendars are de-facto examples of (at least) three key issues when talking about time:

- $\quad$ positioning a time slot inside a continuum,

- handling alternative cycles, granularities, rhythms,

- ordering, comparing, analysing time slots and the way they are depicted here and there, and trying to make sense out of it.

In this contribution, we will focus on historic calendars, i.e. calendars that have been introduced at sometime in the past, have evolved (Julian to Gregorian for instance), ceased to be used (Coligny Gaulish for instance), or remained unchanged (Chinese calendar for instance). The word "historic" might be here a bit confusing, however we have chosen it in order to insist on two aspects that are of importance:

- Handling calendars that are not used any more implies to some extent handling something known to us through testimonies, (i.e. questionable data sets, and accordingly implies confidence assessments).

- Handling calendars that have succeeded to one another - may we know of it or not - implies highlighting parenthoods on one hand (Babylonian - Greek; Egyptian - French Republican) 
and on the other hand calls attention to the consistency of dating (shift of the new year in the Roman calendars).

Let us illustrate a classic implication of this last point with some well-known example:

To honour the date of death of Cervantes, Shakespeare and De la Vega, who supposedly died on the same day, April 23, 1616, UNESCO established April 23rd as the International Day of the Book (UNESCO 2011). But Shakespeare died on a different day than Cervantes and de la Vega - the date of his death (April 23, 1616) is given according to the Julian calendar, and not in the Gregorian calendar, already adopted by Spain but not by England in 1616. Since at that time the Gregorian calendar was ten days ahead of the Julian, the Spanish authors actually died ten days earlier than Shakespeare, whose date of death according to the Gregorian calendar was May 3, 1616. In other words, we are officially asked to remember a fact - three main authors dying the very same day - that never occurred, because of a ten days shift in calendars.

\subsection{From calendars to architectural changes?}

Calendars are an interesting topic by themselves, but what is the connection with architectural changes? Why should we "start from the globe" - time in calendars - when what we are interested in is analysing the evolution of pieces of architecture?

The first reason is a very simple one: there is a clear relation of calendars to architecture. A well known example is the iterative reconstruction of the Temple in Ise (Japan), every 20 years - and 20 years is precisely the Japanese calendar's main cycle (along with eras, connected with the country's rulers). Another simple example is the orientation of Christian churches and chapels in the medieval period. These edifices are usually dedicated to a given saint, and when possible oriented with the apse towards the East at sunrise on the Saint's day, a clear dependence of architecture to the Christian calendar.

The second reason why we chose to "start from the globe" results from an analysis of our previous chronology assessment contributions (Dudek and Blaise 2008, 349-357), (Dudek and Blaise 2011, 632-641), (Blaise and Dudek 2010, 91-100). We have introduced in the past years a number of visualisations aimed at facilitating analytical reasoning. Yet these visualisations rely on a single and common modelling choice, namely - using Aigner's terminology - a one-year chronon time granularity.

And, although we did investigate ordinal time rather than discrete time (Dudek and Blaise 2011, 632641), branching time rather than ordered time (Blaise and Dudek 2011, 8) we are far from having investigated in a thorough way the temporal info we have about architectural changes. And this relative thinness is neither a choice nor an accident. It is the direct consequence of how time is viewed in the context of historical data sets where it is basically seen as a dating issue. Once you have said "circa $I^{s t}$ half of the XIVth century" you're done with the temporal aspect. And so we believe that, if we are to further investigate the time parameter we need to handle historic data sets that go beyond dating - and calendars are well suited to that need.

We expect that developing better tools to model and visualise temporal aspects of historic calendars will help us to re-read our data sets and, for instance, to uncover similarities and differences, constructive innovations, etc..

\subsection{Issue and content}

What does it take to analyse similarities and differences between calendars? It naturally requires a modelling effort, through which common features can be pointed out. Poincaré wrote ... it is in the relations alone that objectivity must be sought, it would be vain to seek it in beings considered as isolated from one another... (Poincaré 1902)

In a way this contribution's main issue could be seen as finding a mean to compare in an insight gaining manner historic calendars. Because calendars were designed that way, modelling will be done by extension - with a subset of 25 calendars chosen (Section 3.1). Furthermore, we will need to integrate confidence assessments in cases where the data is questionable. Given a predefined set of descriptors for each calendar, we will need to develop visualisations in order to sum-up visually a specific calendar's descriptors, or to compare it to others (Section 3.2). Finally, we will need to check out whether or not the proposed framework helps uncovering parenthoods (preferably unexpected otherwise the whole effort might have been vain). 
An exploratory transfer of the calendar framework to the handling of multiple time granularities on architectural cases will be proposed in section 4. Remaining implementation challenges and perspectives of this research will be discussed in section 5 .

One point has to be made clear here: this research is not about the parameter time in general, but about how time is handled inside calendars - i.e discrete time, with a one-day chronon.

Result expected is a set of visualisations helping us to analyse where and when historic calendars have been used, in what they compare to one another. Finally, computational platforms helping users to translate 21st April 2023 (Gregorian calendar) into Chinese, Muslim, Hebrew or other calendars already exist, this aspect will therefore not be mentioned (see for example (Cultural-China 2010), (Philosophia Islamica 2009), (Hebcal Jewish Calendar 2012)) .

\section{Modelling and visualising historic calendars}

As a first step, we initially focused on visual comparison of 25 alternative calendars covering a wide range of historic periods and cultures or civilisations.

(Japanese (Taiintaiyoreki), Tibetan, Babylonian, Burmese, Chinese, Hebrew, Pre-Islamic (Dżahilijja), Symmetry454, Incas (solar), French republican, Egyptian, Soviet, Coptic, Julian, Gregorian, Byzantine, Roman (republican), Inuit, Continental Celtic (Coligny), Muslim calendar (Hijri), Attic state calendar, Maya and Aztec calendars (Xiuhpohualli, Tonalpohualli), Badí', Incas (lunar), and Balinese Pawukon calendars)

\subsection{Modelling}

At first glance a calendar is made of common notions (day, season, year for instance) that need to be adjusted depending on the culture (day finishes with the sunset in the Jewish calendar, there are nine seasons in the Inuit Calendar, year started in November in Gaulish Coligny calendar).

In fact there also are less obvious features that need to be taken into consideration if we are to crossexamine calendars:

- What is the repetitive phenomenon the calendar bases on (sun / moon / seasons, etc.)?

- How are conflicts between natural phenomena (intercalation) solved?

- What granules, are used (i.e. weeks, fortnights, months)?

- What significant cycles are integrated in the calendar?

The grid of descriptors we propose combines several layers. The table below sum up what notions we handle at this stage.

\begin{tabular}{ll} 
Layer & Definition of the descriptor \\
\hline Nature of Temporal Units & phenomenon that defines length of a common year \\
\hline Temporal Units & chronon used (the smallest and indivisible temporal unit) \\
\hline Temporal Units & granules used \\
\hline Temporal Units & $\begin{array}{l}\text { originating position of temporal scale (ex. ab Urbe condita - traditionally } \\
\text { dated to 753 BC) }\end{array}$ \\
\hline Temporal Units & intercalation mechanism (leap year) \\
\hline Temporal Units & episodes (varying granules) \\
\hline Cultural layer & cycles present in calendar \\
\hline Cultural layer & zone of usage (geographical area) \\
\hline Cultural layer & period of validity \\
\hline Thresholds & beginning of year \\
\hline Thresholds & beginning of a day \\
\hline Thresholds & beginning of time countdown \\
\hline Reliability & says to which extent an information is trustworthy \\
\hline Visualisation layer & showing inconsistency (disagreement concerning facts (ex. dates) or their \\
\hline Visualisation layer & interpretation ) \\
\hline Computation layer & showing incompleteness of available information \\
\hline Computation layer & time points \\
\hline Visual layout layer & time intervals \\
\hline Visual layout layer & linear vs. Circular \\
\hline
\end{tabular}


In the following section we present how these notions are actually transferred into graphics (visual cues used), and evaluated on real cases. It has to be made clear that initially we focused on the graphics - we needed to check out whether the whole idea of starting from the globe could make sense. So the implementation we propose is dynamic but poorly structured - script interpretation. Among perspectives of this research is to develop a robust OO-based structure to represent the notions we have identified here above.

\subsection{Visualising}

The first visualisation we propose sums up in a synthetic way fifteen key descriptors of calendars (Fig. 1) distributed inside six visual components (Fig. 2).

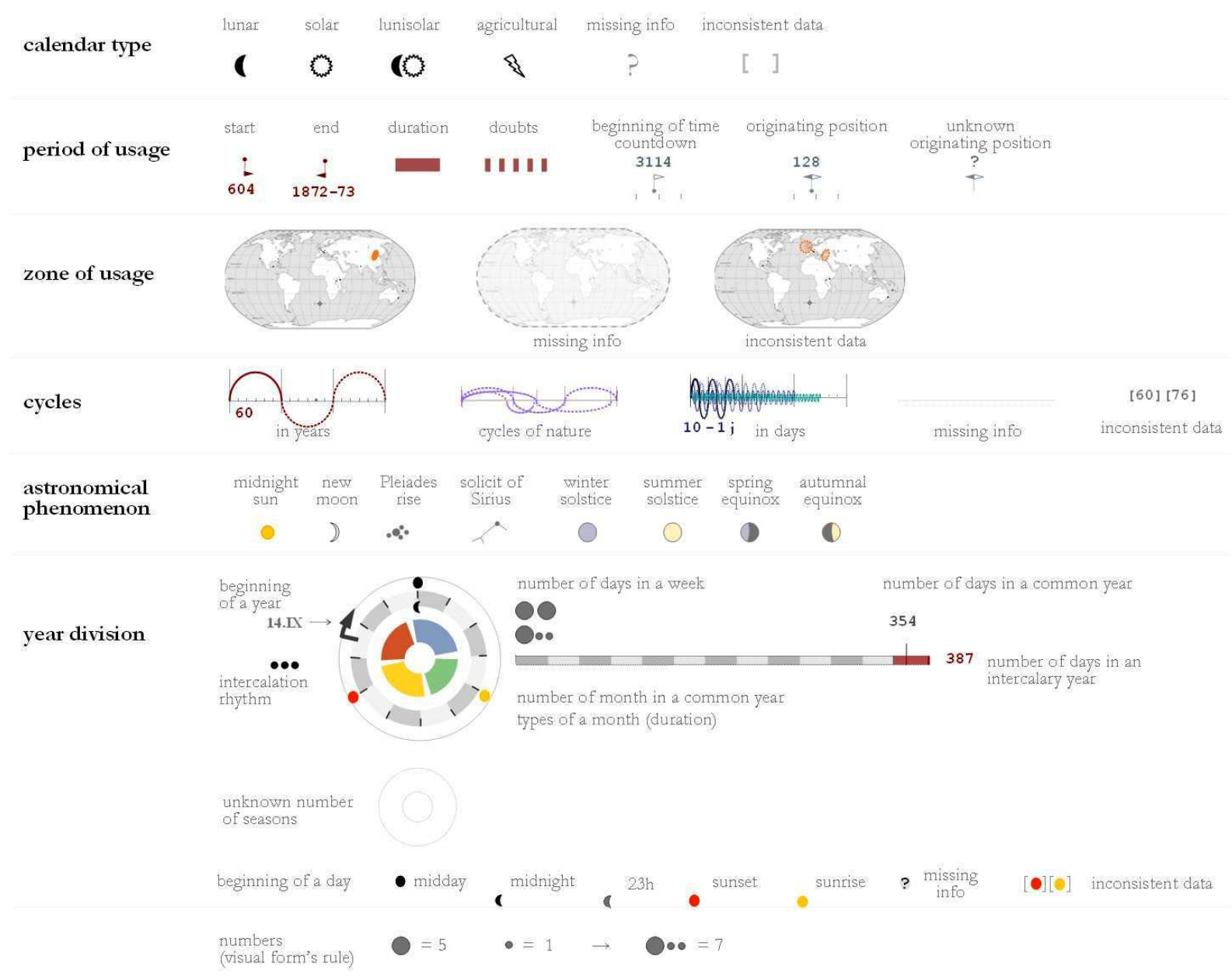

Fig. 1 Visual encoding of the key descriptors taken into consideration in the comparison of calendars.

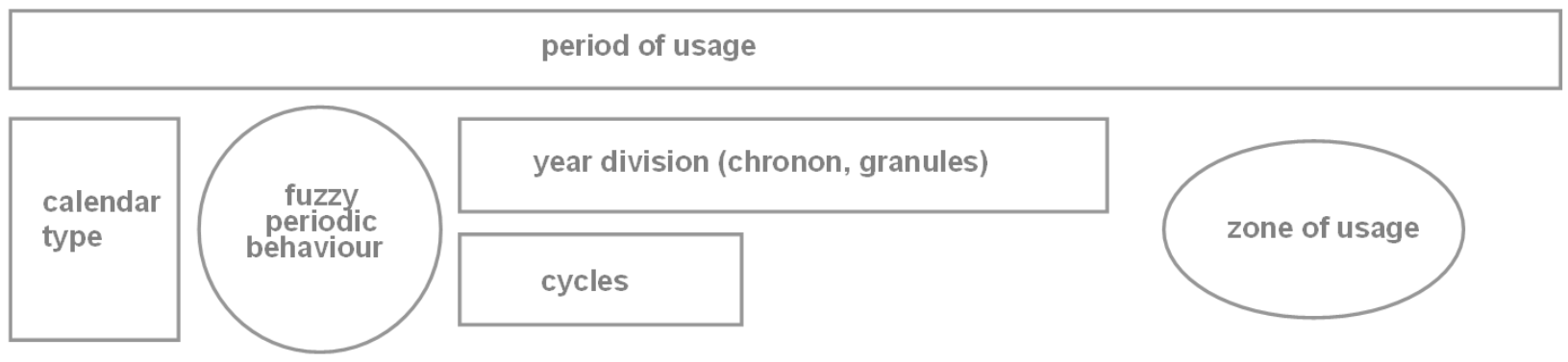

Fig. 2 Distribution of the fifteen key descriptors in graphic components .

The most widespread calendar system today is the Gregorian calendar (solar) introduced in by Pope 
Gregory XIII in a papal bull signed on 24 February 1582. It was adopted in turn by different countries. Gregorian calendar modified the Julian calendar's regular cycle of leap years (leap years are exactly divisible by four except for years that are exactly divisible by 100 , but the centurial years that are exactly divisible by 400 are still leap years). A Week is the smallest granule (7 days). Each year counts 12 months (365 days in common years, 366 days in leap years). Beginning of a year was maintained on 1st January.

Until the adoption of a resolution of the International Meridian Conference, each town used its own local time. Lengths of each day-time hour and night-time hour were unequal and depended on the geographical latitude and varied according to the seasons - what is more a day could begin at noon, sunrise or sunset, reflecting the wide diversity of customs for defining and counting the hours across the European continent (Fig. 3).

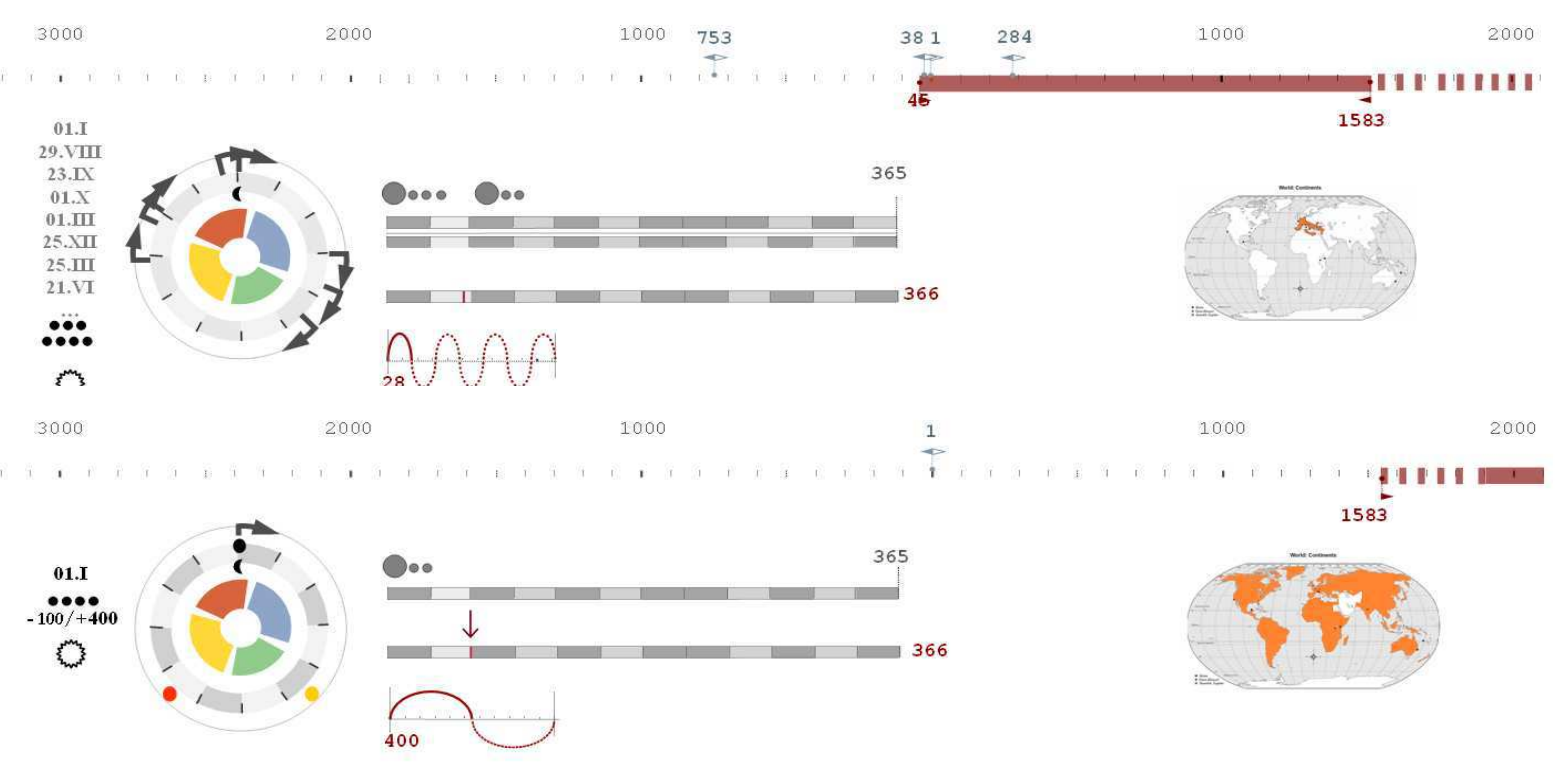

Fig. 3 Visual comparison of the Julian and Gregorian calendars

Influence of a culture and climate is plainly visible in Inuit calendar. The absence of a notion of day or week in Inuit's culture, as well as surprising number of seasons (related to the cycles of nature). On the other hand the French republican calendar has a clear "goddess reason" decimal structure (Fig. 4).

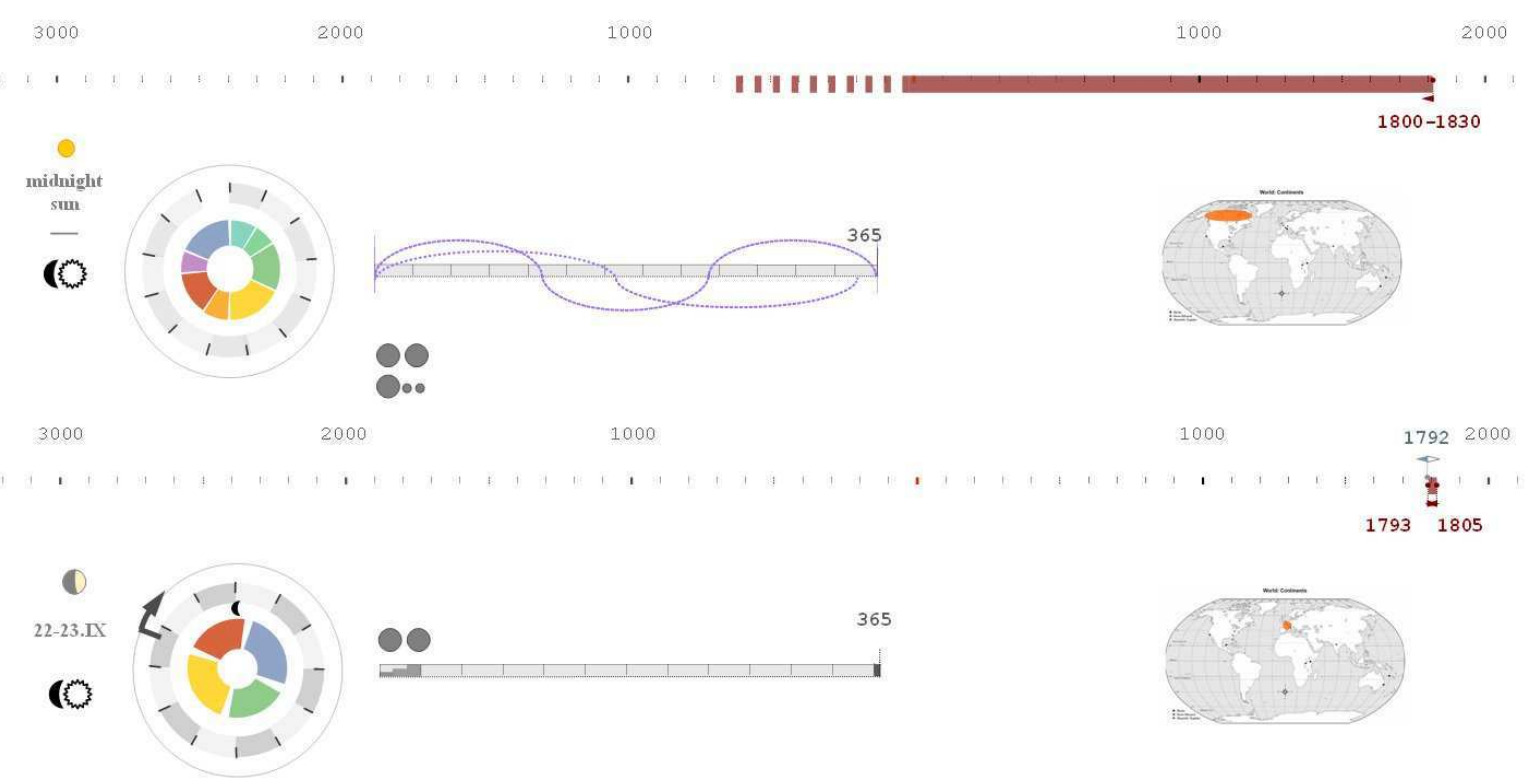

Fig. 4 Visual comparison of the Inuit and French republican calendars 
In order to foster comparison of the whole collection (Fig. 5), we developed a second visualisation underlining:

- legacies in between periods and areas (e.g. The Coptic Year is the extension of the ancient Egyptian civil year retaining its subdivision into the three seasons.),

- presence of cycles, alternative divisions of the year (e.g. Agriculture related calendars Balinese Pawukon calendar or Incas agricultural calendar - seem quite weird compared to a calendars based on a sidereal year.),

- mechanisms to cope with intercalations, etc.

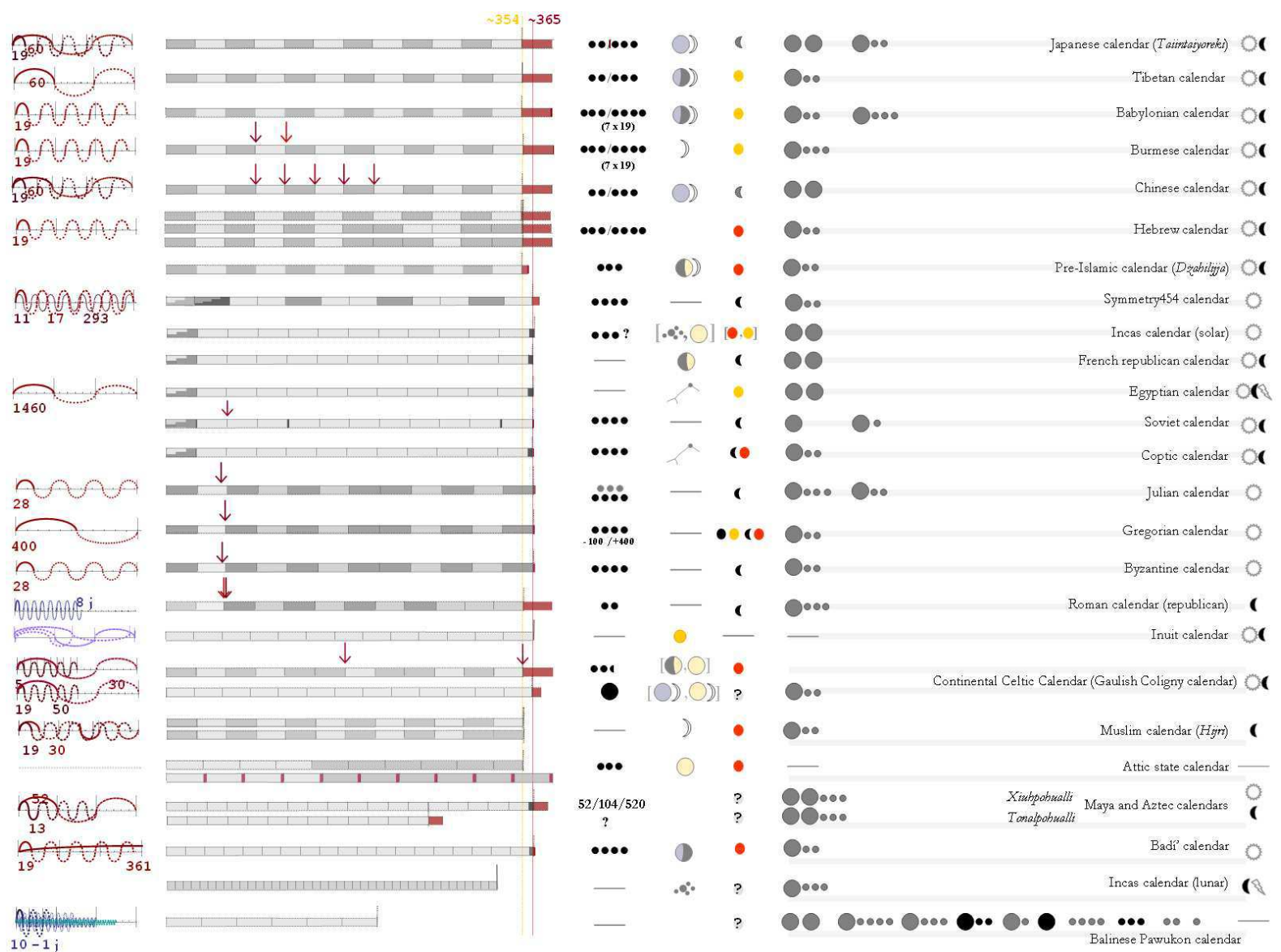

Fig. 5 A visual comparison of 25 calendars

This comparison allowed us to ascertain similar mechanisms of time discretisation - day plays role of chronon in an overwhelming majority of cases and it is aggregated into widely used, standard granules (i.e. week, month, season, year, century) or some less employed ones (e.g. days outside a year). However the exact number of days in a given granule substantially differs (e.g. 12 months in a common year is not a rule but only a dominant trend).

Moreover beginning of time countdown and originating position of temporal scale strongly varies. These parameters as well as intercalation mechanisms, beginning of a year or a day are culture dependent - showing us more about societies and their way of life than about the time.

A very common and instructive parameter is periodicity of calendars - presence of natural or calendar based cycles. Once again, cultural based cycles (e.g. ruler's lifetime, market day's cycle, unlucky day's cycle, intervals associated with different groups of deities, ...) throws light on humans and societies, zones of influences helping us in teleological reasoning, etc...

Natural cycles (climate or astronomical related) although profoundly built in calendar structure are independent from the calendar structure - even in most precise calendars they move slightly inside a sequence of a year. This relative freedom of natural cycles is reinforced by a recurrent phenomenon: a discontinuity of time in calendars due to human manipulation - time in calendars is stretched or shortened from time to time (e.g. 80 additional days in year 46th BC, 11 days less in 1582 AD a.s. in Madrid and Cordoba, but not in Stratford-upon-Avon or in Southampton).

This first result has been extended to propose a more generic framework for visualising time with 
multiple granularities. It is applied on two different test cases: chapel of St Anne in Southern Alps (France) and the belfry of Cracow's former town hall in (Poland).

\section{Visualising time-oriented historical data}

Naturally the visualisation of time-oriented data poses various types of difficulties, with a number of them generating diverse types of uncertainties:

- modelling choices of temporal variables,

- data credibility (i.e. heuristic accuracy and bias of analyst),

- inconsistency of data - that implies possibility of various scenarios,

- incompleteness of data,

- subjectivity of choices (amount of interpretation or personal judgment included),

- approximations and imprecision in data description (e.g. How to interpret expressions like probably in 1567?),

- temporal resolution of data - that relates to temporal granularity,

- etc.

All these elements should be integrated into any visualisations to improve the cognitive task of spatiotemporal understanding. In this paper we will limit our discussion to the last point that relates to time granularity.

We will start with a brief introduction into problems posed by visualisation of datasets characterised by varying temporal granularity. In a following section we will present a proposal framework of a method of visualising time with multiple granularities.

\subsection{Visualisation of datasets characterised by varying temporal granularity}

Historical data sets are not consistent in terms of temporal granularity. In other words the individual temporal resolution of pieces data of may vary.

Let's take as an example three different pieces of information concerning the same object:

A fire in the town hall's tower took place in summer 1543.

A modification of a tower started on 15th August 1543.

A big quantity of bricks have been bought in August 1543.

Each piece of information has its own temporal granularity. If the chronon of our visualisation (the smallest unit) is one civil year (date format YYYY), information about the events that occurred in the same year can be represented only as a group of events that took place in 1543 . What is more other hints about temporal relations between these events (i.e." in summer" contains "in August" contains "15th August") will disappear.

If our choice for the chronon is one day (date format DD MM YYYY), granularity of the visualisation will exceed the natural temporal granularity of the underlying information. In such situations the description and visualisation of events compels to introduce approximated, interpretation-derived values, thereby reinforcing the global uncertainty on temporal aspects (i.e."in summer" does no mean each and every summer day). In other words, whatever chronon we choose, and represent, there will be pieces of information that just don't fit - either they are too "thin" and the foot moves in the shoe, or too big and the foot is shrunk to fit in the shoe.

Accordingly, and taking advantage of what we had observed on the variable granularity of calendars and on the importance of fuzzy periodic comportment of seasons, we have tried to support timeoriented reasoning tasks with mechanisms that would display in a unique visual layout several granularities at a glance. The visual formalism, first experimented on calendars and seasons, provides a simple solution to handle both a day granularity and more fuzzy intervals within a cyclic-time enhancing visualisation (Fig 6).

It is in fact a rather generic formalism (it could be applied to year and centuries, or to any other combination) that bases on two principles:

- concentricity on one hand - mechanism to segregate granularities,

- sector vs. point on the other hand - mechanism to allow switches from chronon to granules, including fuzzy granules. 

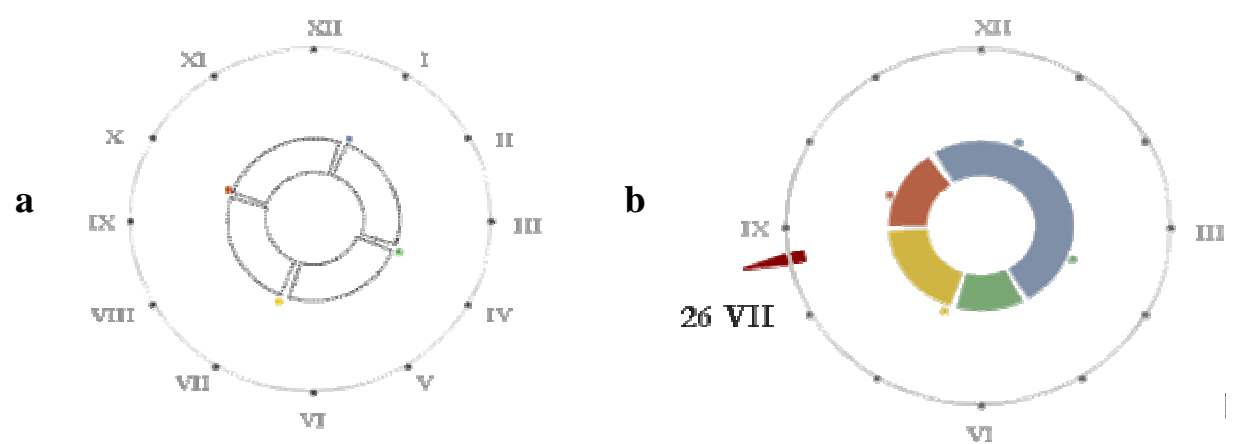

Fig. 6 Cyclic time visualisation mode exploits a 'clock' metaphor - one o'clock stands for beginning of January. Beginning of astronomical seasons is marked by colour dots and meteorological seasons are represented by coloured sectors ('rose of seasons') - they may not coincide (b)

\subsection{Impact on representing architecture changes}

The first case study is Ceillac's chapel of St Anne, in Southern Alps (altitude $2400 \mathrm{~m}$ ).

The cyclic events related to this artefact include an annual pilgrimage to the chapel (July 26th) and naturally the cycle of seasons (Fig 7, a). At this altitude meteorological seasons do not coincide with astronomical seasons. Winter is the longest season here but its length may vary (season's length has a fuzzy periodic behaviour), therefore it is important to point out that in our cyclic events representation we show values for an average season length - variations of this factor has to be taken into consideration by analysts.

The information about the chapel of St Anne was gathered and represented using a classic timeline with a granularity of one year (Fig 7, b), but it is not the natural temporal resolution of all the data we dispose. Pieces of information we dispose are dated with a variable precision (e.g. DD/MM/YYY, MM/YYY, season YYYY, YYYY). Increasing the time granularity (e.g. operating with a day) makes a visualisation impractically long, what is more it introduces the unavoidable problem "shrinking the foot to fit in the shoe" with interpretation-derived values.

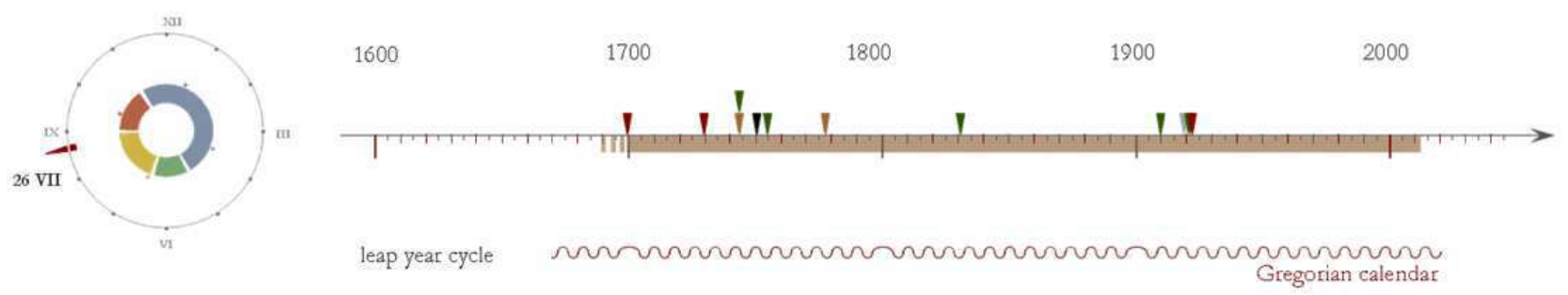

Fig.7 St Anne chapel a) cyclic time visualisation mode; b) timeline - Different colours mark different types of events. The overall evolution of the chapel is marked with the brownish line. All known transformations were described according Gregorian calendar.

Our proposal is to use at visualisation time the natural temporal granularity of the dating associated with events (Fig 8).

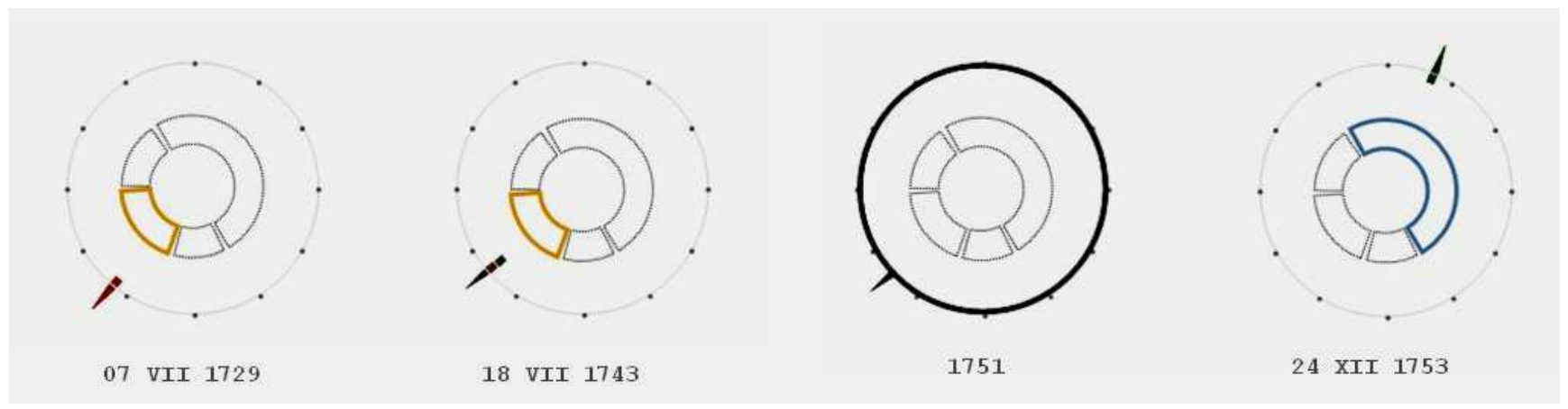

Fig.8 St Anne chapel - Events dating represented with their natural, heterogenesous; temporal granularity. From left to right : DD/MM/YYYY format, MM/YYYY format, season + YYYY format, and YYYY format. 
This type of visualisations may be reorganised according to additional criterion (e.g. by events type) in order to help in reasoning. It is also possible to produce them for a selected time span (e.g. two decades, a century or a whole life of an artefact (Fig 8).

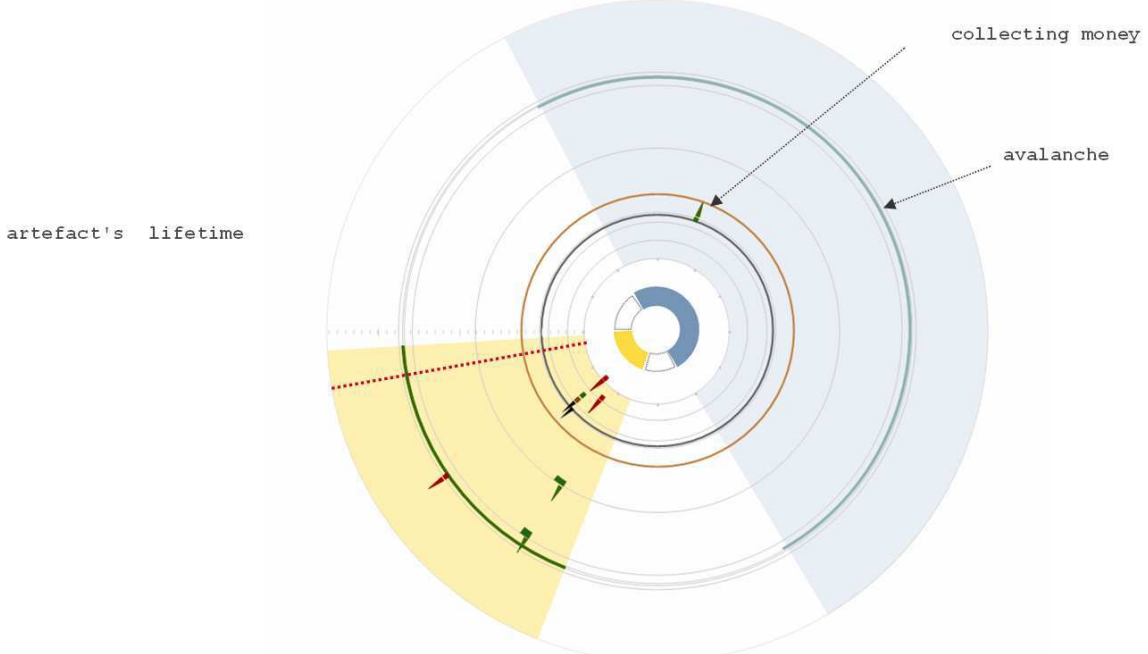

Fig 8 Visualisation produced for a whole life of the artefact. Note that all the events related to the chapel took place in summer (i.e. before st Anne's day) except of a collect of the money and avalanche - a fact very easy to consider as obvious (altitude $2400 \mathrm{~m}$ ) once the visualisation did the job for us...

Linear and cyclic visualisation modes underline different particularities of the data sets: it should be stated clearly that their combination notably reinforces their power of assistance in reasoning. What this simple experiment shows is not that one is better than the other - the basic linear timeline is for instance very useful in underlining temporal densities - but digging into that temporal aspects requires to think outside the square in terms of visualisation.

The second case study is the belfry of Cracow's former town hall, and more precisely its fires.

It is not trivial to note that two case studies - chapel of St Anne and the belfry of Cracow's former town hall - have not the same location, therefore time measure (different calendars) and climatic conditions are different (Fig 9a). The lifetime of the belfry traverses two calendars and it had some relation with the seasons that have been moving inside year cycle (we present only a rough approximation of the changes).

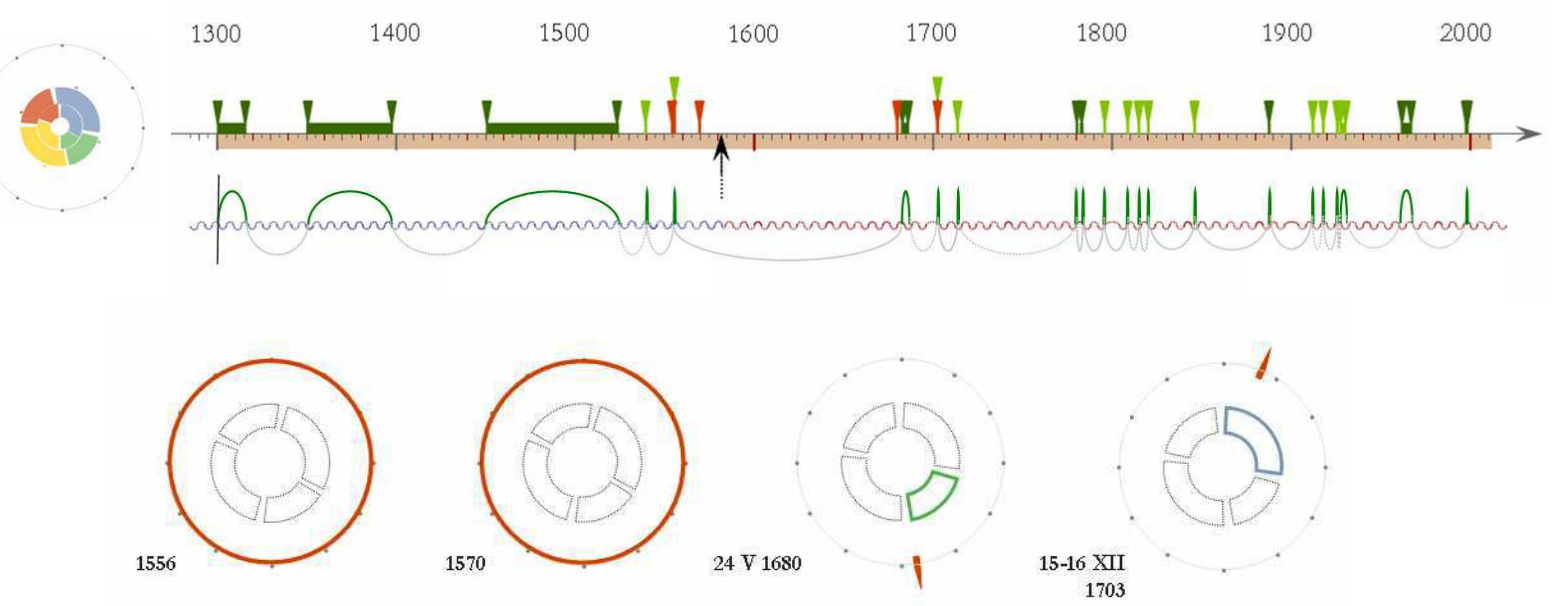

Fig 9 The belfry of Cracow's former town hall. The classic timeline indicates intervals of transformations (green colour) and fires of the artefact (orange colour).

a) average meteorological seasons do not coincide with the astronomical seasons, summer is the longest season in Cracow, no cyclic event for this artefact has been found 
b) rhythm of artefact transformations/ rhythm of a leap year

c) dates of known fires of the town hall tower with their specific temporal resolution

Although we managed to visualise all dates of known fires of the artefact with their specific temporal resolution, it is hard to find any regularity basing our reasoning on four cases only. One could be tempted to say that: the oldest historical sources are less precise in terms of dating.

We have therefore verified this hypothesis on the ensemble of buildings that furnished the entire Main Market Square in Cracow (Fig 10) and it turned out to be false. Unsurprisingly, we notice no regularity, no cyclic comportment inside these datasets.

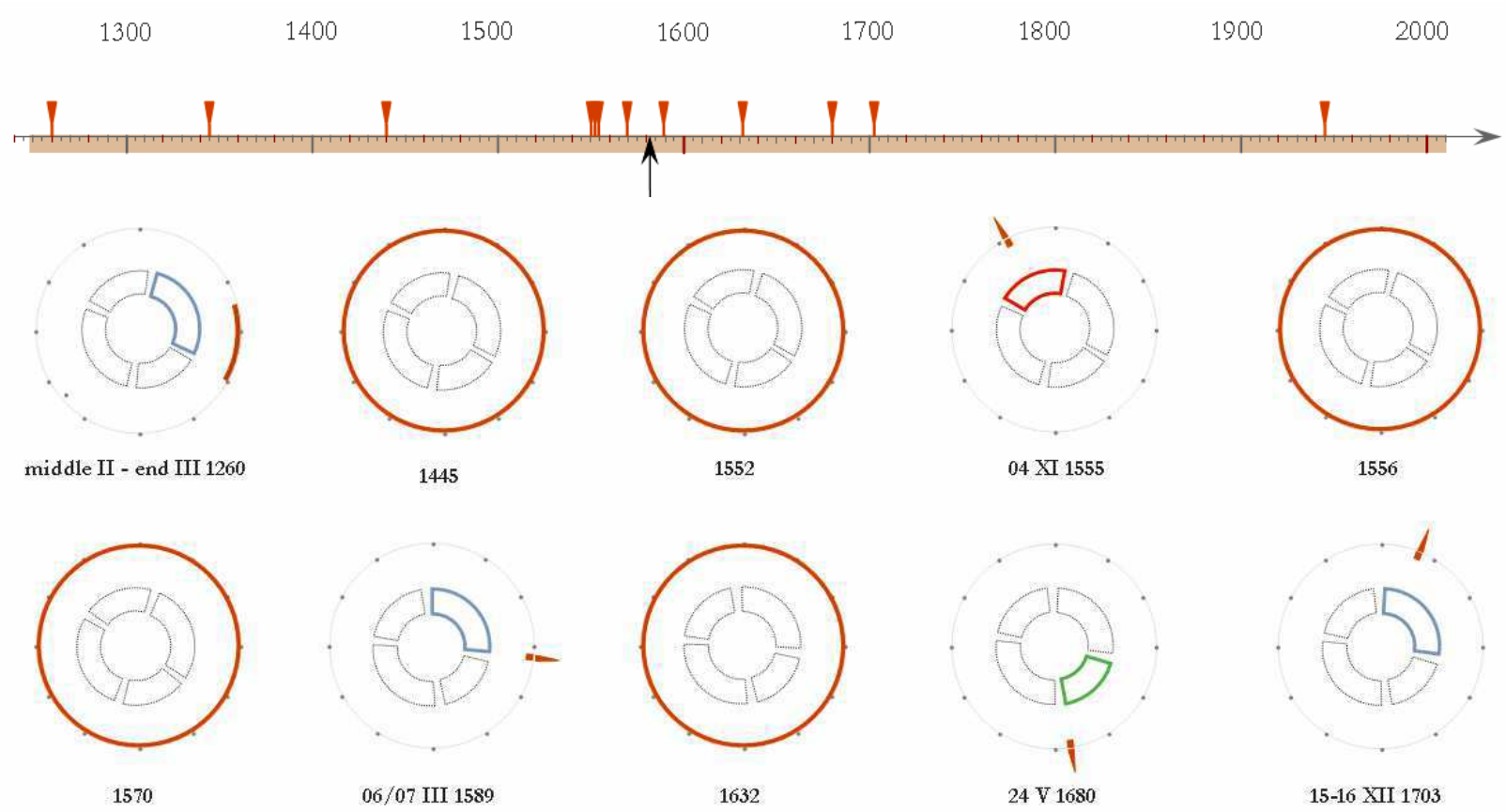

Fig 10 a timeline listing all known fires of the ensemble of buildings that furnished the entire Main market Square in Cracow - a fire in town is a punctual phenomenon.

\section{Conclusions}

This contribution presents bases of a method we have tried to develop in order to visualise time with multiple granularities, in order to support reasoning on various aspects of temporal relations (cyclic or fuzzy periodic comportments) in the context of heterogeneous temporal data sets. In short, this experience was primarily about "shaking the tree" of possibilities when one needs to handle multiple granularity.

At the current stage of development we wish to restrict our conclusions to the following remarks:

- properly designed visualisation helps in reasoning (visual comparisons, visual thinking)

- study of cyclic and fuzzy periodic behaviour may help in better understanding data sets we handle in the context of historic sciences,

- periodic behaviour analysis requires specific visual tools (beyond basic linear timelines),

- differently localised artefacts may necessitate different visual instruments - 'tailoring' to the local conditions (in particular to the succession of calendars with various - sometimes local time discontinuities, cycles of seasons, time granulation, etc.),

- if we deal with the notion of time in our research, it is worth to take some time to think about "time" for itself, and not about time as a date stamp we need to put on a given piece of data.

It has to be said clearly that further work, especially in terms of implementation, is necessary if we wish to further investigate potential benefits and drawbacks of this approach. Yet there is one lesson that probably deserves to be drawn in conclusion: although most often a risky bet, starting from the globe - what we did by starting from calendars in order to depict temporal aspects of architectural changes - may be a fruitful attitude sometimes; in particular when talking about things we think we 
know well. Talking about time, Saint Augustin said: "if no one asks me [what is time], I know. But if I wanted to explain it to one who asks me, I plainly do not know". Those who investigate the time parameter probably tend to think he was not that wrong - the issue remains today a challenging one, particularly in the context of historic sciences.

MacEachren, Alan M., Mark Gahegan and Elisabeth Hetzler. 2005. "Visualizing geospatial information uncertainty: What we know and what we need to know", Cartography and Geographic Information Science 32/2: 139-160.

Tufte, Edward. R. 1997. Visual Explanation. Cheshire: Graphics Press

Aigner, Wolfgang., Silvia Miksch, Wolfgang Müller, Heidrun Schumann and Christian Tominski. 2008. "Visual methods for analysing time-oriented data" in IEEE Transactions on Visualization and Computer Graphics 14,/1. Citeseer: 47-60.

Knight B and J. Ma. 1993. "Time representation: A taxonomy of temporal models" Artificial Intelligence Review 7/6: 401-419

Tufte, Edward. R. 1990. Envisioning Information, Cheshire: Graphics Press

Matoušek, Kamil., Martin Falc and Zdeněk Kouba. 2007. "Extending Temporal Ontology With Uncertain Historical Time" Computing and Informatics 26: 239-254

Allen, James.F. 1984. "Towards a general theory of action and time" Artificial Intelligence 23: 124154

Peralta, Duarte Nuno, H. Sofia Pinto and Nuno J. Mamede. 2004. "Reusing a Time Ontology" In Enterprise Information Systems V , ed. Camp, Olivier., Joaquim B. L. Filipe, Slimane Hammoudi, And Mario Piattini, 241-248, Springer Netherlands.

West, Ken. 2010. "Theory vs. practice" The News \& Advance, September 27.

Accessed Mai 28, 2012. http://www2.newsadvance.com/lifestyles/2010/sep/27/theory-vs-practice-ar$528499 /$

Chardonnel, Sonia. 2007. "Time-Geography: individuals in time and space." In Models in Spatial Analysis, ed. Lena Sanders, London: ISTE, doi: 10.1002/9780470612255.ch4

Dudek, Iwona and Jean-Yves Blaise. 2008. "Profiling artefact changes: a methodological proposal for the classification and visualisation of architectural transformations.", In Proceedings of VSMM 2008, ed. M. Ioannides, A. Addison, A . Georgopoulos, and L. Kalisperis, 349-357, Budapest :

Archeolingua.

Blaise, Jean-Yves and Iwona Dudek. 2010. "Understanding changes in historic architecture. Can we provide tools \& methods for visual reasoning?", In Proceedings of International Conference on Information Visualization Theory and Applications, ed. Richard Paul. and Braz José., 91-100. INSTICC Press.

Dudek, Iwona and Jean-Yves Blaise. 2011. "Concentric Time: Enabling Context + Focus Visual Analysis of Architectural Changes", In Foundations of Intelligent Systems, ed. M. Kryszkiewicz, H. Rybinski, A. Skowron, W. Raś, 632-641. Berlin, Heidelberg : Springer-Verlag.

Blaise, Jean-Yves and Iwona Dudek. 2011. "Visualizing alternative scenarios of evolution in heritage architecture", In Proceedings of the 11th International Conference on Knowledge Management and Knowledge Technologies, ed: Stefanie N. Lindstaedt, Michael Granitzer, New York, ACM International Conference Proceeding Series ACM.

Poincaré, Henri. 1902. La Valeur de la Science, http://fr.wikisource.org/wiki/La_Valeur_de_la_Science 
cultural-china. 2010. "Gregorian Calendar- Chinese Lunar Calendar Converter." Accessed February 192012.

http://www.cultural-china.com/Kaleidoscope/cultural_tool/Calendar_Converter/index.html

Philosophia Islamica. 2009. "Conversion of Hijri A.H. (Islamic) and A. D. Christian (Gregorian) dates. " Last modified October 132009.

http://www.muslimphilosophy.com/ip/hijri.htm

Hebcal Jewish Calendar. 2012. "Hebcal Jewish Calendar.” Last modified April 022012.

http://www.hebcal.com/converter/

UNESCO. 2011. "World Book and Copyright Day."

Accessed February 192012

http://www.unesco.org/new/en/unesco/events/prizes-and-celebrations/celebrations/internationaldays/world-book-and-copyright-day-2012 